\title{
A retrospective study of the occurrence of ischemic-type biliary lesions post liver transplantation
}

\author{
Rui Shi, Ya-Min Zhang ${ }^{*}$, Yong-Lin Deng, Hong Zheng, Cheng Pan \& Zhong-Yang Shen \\ Tianjin Key Laboratory of Organ Transplantation, Department of Hepatobiliary Surgery, First Center Hospital Clinic Institute, Tianjin \\ Medical University, Tianjin 300192, China
}

Received November 12, 2015; accepted December 26, 2015; published online April 21, 2016

Citation: Shi, R., Zhang, Y.M., Deng, Y.L., Zheng, H., Pan, C., and Shen, Z.Y. (2016). A retrospective study of the occurrence of ischemic-type biliary lesions post liver transplantation. Sci China Life Sci 59, 529-531. doi: 10.1007/s11427-016-5041-6

\section{Dear Editors,}

Ischemic-type biliary lesions (ITBLs) significantly reduce patient and graft survival rates. Research data regarding the causes of ITBL are rare. ITBLs is an important cause of post liver transplantation graft loss and patient death. The exact cause of this type of biliary complication remains unclear. To establish strategies for ITBL treatment or prevention, the risk factors of ITBL must be elucidated. The objective of this single-center retrospective study was to investigate the risk factors of ITBL in orthotopic liver transplantation (OLT) patients.

ITBLs occur in liver transplant recipients with symptoms of biliary stenosis, expansion, biliary abscess formation, biliary disappearance, and liver dysfunction. The reasons for this occurrence remain unknown. However, some possible reasons have been proposed, such as hepatic artery thrombosis, antibody-mediated rejection, primary sclerosing cholangitis, or chronic rejection, which may cause similar symptoms but are not included as causes of ITBLs.

In this retrospective study, 1,328 OLT patients with complete data who underwent liver transplantation between August 2007 and January 2014 at the Tianjin First Central Hospital Organ Transplant Center were considered for the study. In accordance with the definition of ITBL, we used the following exclusion criteria: (i) postoperative hepatic arterial thrombosis; (ii) ABO blood group incompatibility

*Corresponding author (email: zhangyamin@medmail.com.cn) liver transplantation; (iii) a preoperative diagnosis of primary sclerosing cholangitis; (iv) patients with postoperative chronic rejection; and (v) a survival or follow-up time of $<3$ months after transplantation. The data of 886 OLT patients were included in the study.

The statistical calculations were performed with the SPSS 19 software (IBM, USA). In this study, $P$ values of $<0.05$ were considered statistically significant.

The data included the following primary disease statistics: 530 cases of hepatitis B cirrhosis; 109 cases of hepatitis $\mathrm{C}$ cirrhosis; 33 cases of non-hepatitis malignant hepatic tumor; 55 cases of alcoholic cirrhosis; 42 cases of primary biliary liver sclerosis; 33 cases of cryptogenic cirrhosis; 31 cases of autoimmune hepatitis; 27 cases of retransplantation, 15 cases of congenital disease; two cases of Budd-Chiari syndrome; three cases of drug-induced liver disease; two cases of polycystic liver; and four cases of Wilson's disease.

The overall ITBL incidence in this group of patients was $14.00 \%(124 / 886)$.

The donor- and surgery-related risk factors are shown in Table 1. The risk factors include cold ischemic time, non-hepatic phase, amount of intraoperative red blood cells used, and the amount of intraoperative blood plasma used. The data were analyzed by using an independent sample $t$ test. The classification data were analyzed with a cross-tabulation test.

The SPSS 19 software (IBM company spss19, USA) was used in the multivariate analysis. All 886 cases were included in the multivariate analysis by using a logistic re- 
Table 1 Donor, recipient and surgery-related risk factors

\begin{tabular}{|c|c|c|c|c|c|}
\hline Group & Cases & Non-ITBL & ITBL & Statistics & $P$ \\
\hline Cases, $n$ & 886 & 762 & 124 & - & - \\
\hline Donor factors & - & - & - & - & - \\
\hline Donor age & - & $33.84 \pm 9.75$ & $34.63 \pm 9.63$ & $t=0.835$ & 0.763 \\
\hline Cold ischemia (min) & - & $486.88 \pm 210.41$ & $572.98 \pm 174.53$ & $t=4.320$ & 0.000 \\
\hline Graft weight (g) & - & $1003.64 \pm 293.38$ & $1066.48 \pm 225.20$ & $t=2.278$ & 0.023 \\
\hline Donor steatosis & - & - & - & - & - \\
\hline None & 790 & 680 & 110 & $\chi^{2}=0.67$ & 0.712 \\
\hline Mild <30\% & 82 & 71 & 11 & - & - \\
\hline Moderate $30 \%-60 \%$ & 14 & 11 & 3 & - & - \\
\hline Storage solution & - & - & - & - & - \\
\hline $\begin{array}{c}\text { University of Wisconsin organ perfusion } \\
\text { preservation solution }\end{array}$ & 788 & 673 & 115 & $\chi^{2}=2.21$ & 0.092 \\
\hline Histidine-tryptophan-ketoglutarate & 98 & 89 & 9 & - & - \\
\hline Recipient factors & - & - & - & - & - \\
\hline Recipient sex & - & - & - & - & - \\
\hline Male & 726 & 619 & 107 & $\chi^{2}=1.84$ & 0.208 \\
\hline Female & 160 & 143 & 17 & - & - \\
\hline Recipient age & - & $48.03 \pm 15.69$ & $51.14 \pm 9.12$ & $t=1.936$ & 0.053 \\
\hline Blood type & - & - & - & $\chi^{2}=5.860$ & 0.119 \\
\hline A & 246 & 212 & 34 & - & - \\
\hline $\mathrm{B}$ & 281 & 232 & 49 & - & - \\
\hline $\mathrm{AB}$ & 112 & 96 & 16 & - & - \\
\hline $\mathrm{O}$ & 247 & 222 & 25 & - & - \\
\hline Model of end-stage liver disease score & - & $16.54 \pm 8.66$ & $16.64 \pm 9.55$ & $t=0.109$ & 0.914 \\
\hline Child-Pugh & - & - & - & $\chi^{2}=1.092$ & 0.579 \\
\hline A & 232 & 201 & 31 & - & - \\
\hline $\mathrm{B}$ & 363 & 307 & 56 & - & - \\
\hline $\mathrm{C}$ & 291 & 254 & 37 & - & - \\
\hline Primary disease & - & - & - & $\chi^{2}=4.088$ & 0.770 \\
\hline Hepatitis B virus & 530 & 447 & 83 & - & - \\
\hline Hepatitis C virus & 109 & 96 & 13 & - & - \\
\hline Hepatocellular carcinoma (Non-hepatitis) & 33 & 30 & 3 & - & - \\
\hline Primary biliary liver sclerosis & 42 & 36 & 6 & - & - \\
\hline Retransplantation & 27 & 23 & 4 & - & - \\
\hline Autoimmune hepatitis & 31 & 28 & 3 & - & - \\
\hline Alcoholic cirrhosis & 55 & 50 & 5 & - & - \\
\hline Others & 59 & 52 & 7 & - & - \\
\hline Surgery-related risk factors & - & - & - & - & - \\
\hline Non-hepatic (min) & - & $47.44 \pm 15.06$ & $53.98 \pm 22.59$ & $t=4.139$ & 0.000 \\
\hline Red blood cells (unit) & - & $11.81 \pm 11.25$ & $15.06 \pm 11.02$ & $t=2.990$ & 0.003 \\
\hline Blood plasma (mL) & - & $2038.01 \pm 1195.92$ & $2565.73 \pm 1053.98$ & $t=4.629$ & 0.000 \\
\hline Cytomegalovirus infection & - & - & - & $\chi^{2}=0.032$ & 1.000 \\
\hline Positive & 27 & 4 & 12.90 & - & - \\
\hline Negative & 735 & 120 & 14.04 & - & - \\
\hline Acute rejection & - & - & - & $\chi^{2}=1.224$ & 0.229 \\
\hline Occurrence & 9 & - & 25.00 & - & - \\
\hline Non-occurrence & 753 & 121 & 13.85 & - & - \\
\hline
\end{tabular}

gression model. All of the cases were divided into two groups according to the occurrence of ITBL as follows: the ITBL group $(n=124)$ and the non-ITBL group $(n=763)$. The following factors were analyzed: recipient age, model of end-stage liver disease (MELD) score, Child-Pugh classification, cytomegalovirus (CMV) infection status, nonhepatic period, intraoperative red blood cells used, freshfrozen plasma dosage, acute rejection, recipient sex, graft weight, fatty liver degree, and cold ischemic time. The results are shown in Supporting Information. The results showed a statistically significant difference between ITBL occurrence and cold ischemic time $(P=0.000)$, the nonhepatic period $(P=0.002)$, and the amount of intraoperative frozen plasma used $(P=0.012)$. The cold ischemic time, non-hepatic period, and amount of intraoperative frozen plasma used were the independent risk factors of ITBL occurrence.

The clinical data of the 886 liver transplant patients were analyzed in this study. The ITBL incidence was $14 \%$. The reported incidence of ITBL ranged from $1.4 \%$ to $26 \%$ (Zhang et al., 2014). The donor age and cold ischemic time were reported to be significant risk factors of the occurrence of ITBL. ITBL occurs more frequently when the liver is preserved in a histidine-tryptophan-ketoglutarate organ perfusion preservation solution than in the University of Wisconsin organ perfusion preservation solution (Girometti 
and Baccarani, 2013). Some researchers found that the presurgical MELD and Child-Pugh scores of the recipients significantly affect the incidence of ITBL (Wang et al., 2013). CMV infection status and immune factors such as the effect of acute rejection have been reported to be risk factors of ITBL (Wang et al., 2013; Frongillo et al., 2014).

Two views have emerged regarding the factors that determine the occurrence of ITBL after liver transplantation. First, the disease starts during the period in which the donor liver undergoes cold ischemia, warm ischemia, and reperfusion injury; a latency period occurs until the results of biliary disease are manifested (Boyko et al., 2014). Second, early postoperative immune factors invade the donor liver and repeatedly attack the biliary tract, and an ITBL gradually forms (Girometti and Baccarani, 2013).

This study, as well as most studies in the literature, presents evidence that strongly supports the first hypothesis and the theory of damage during preservation (Frongillo et al., 2014). Further research should focus on the mechanism of biliary tract disease that occurs during storage and on intraoperative findings.

In this study, the multivariate analysis revealed that fatty liver and perfusion fluid types did not influence the incidence of ITBL. However, cold ischemic time showed a statistically significant difference. Some studies have shown that $>10$ hours of cold storage time has a significant effect on the incidence of ITBL (Wang et al., 2013). Minimizing the cold ischemic time is probably advantageous to patients. However, given the severe shortage of donors, the risk of death during the waiting period must be considered as well. In recent years, the use of marginal donors, older donors, donor liver steatosis, donor livers with long cold storage time, low-perfusion donor livers, and grafts with hepatitis has increased. In particular, the use of marginal donor organs has become controversial, and the task of ITBL prevention has become more difficult.

In this study, the non-hepatic period is an independent risk factor of ITBL, which is a relatively unique finding. Most studies have concluded that ITBL is not correlated with non-hepatic time, the amount of intraoperative red blood cells used, and plasma use, among other factors. By contrast, in our group of patients, a strong correlation was shown. The above-mentioned results reflect the increasing surgical difficulties that affect the occurrence of ITBL. These indicators are frequently concomitant with prolonged intraoperative graft ischemic time, severe hypotension, liver hypoperfusion, and delayed liver function recovery. Research on the relationship between early graft function and intraoperative conditions to ITBL is rare (Boyko et al.,
2014). To identify the effect of surgical factors on ITBL, surgeons should conduct further research on the relationship between intraoperative factors and ITBL.

Plasma use during operation was found as a risk factor of ITBL occurrence. In the univariate analysis, we found that red blood cell use is also a risk factor. However, in the multivariate analysis, between the two factors, that which showed a relationship with ITBL occurrence was identified. Thus, plasma use during operation was the only independent risk factor identified. Difficulty in operating, poor preoperative liver function, and delayed donor function recovery could lead to additional plasma use during operation. Altogether, these adverse factors could cause the development of ITBL.

This study shows that cold ischemic time, non-hepatic time, and the amount of intraoperative blood plasma used are the main risk factors of ITBL occurrence. These results show that ITBL closely correlates with liver cold preservation and surgical procedures. Efforts to clinically reduce the incidence of ITBL should focus more on shortening liver preservation and surgical times, and reducing blood loss by selecting a more experienced transplant surgery group to complete the operation.

Compliance and ethics The author(s) declare that they have no conflict of interest.

Acknowledgements This work was supported by the National Key Clinical Specialty Construction Project of Organ Transplantation Department (201354415), National High Technology Research and Development Program of China (2012AA021001), National Natural Science Foundation of China (81370576), Tianjin Health Bureau Technology Fund (2013KZ031), and Tianjin health industry key research project (12KG102).

Boyko, V.V., Pisetska, M.E., Tyshchenko, O.M., Skoryi, D.I., Kozlova, T.V., Gorgol, N.I., and Volchenko, I.V. (2014). Role of ischemic preconditioning in hepatic ischemia-reperfusion injury. Hepatobiliary Surg Nutr 3, 179-184.

Frongillo, F., Lirosi, M.C., Sganga, G., Grossi, U., Nure, E., Avolio, A.W., Bianco, G., Mariano, G., and Agnes, S. (2014). Graft steatosis as a risk factor of ischemic-type biliary lesions in liver transplantation. Transplant Proc 46, 2293-2294.

Girometti, R., and Baccarani, U. (2013). Magnetic resonance cholangiopancreatography in assessing living liver donors biliary anatomy: opportunities and challenges. Hepatobiliary Surg Nutr 2 165-167.

Wang, H., Jiang, W., Zhou, Z., Long, J., Li, W., and Fan, S.T. (2013). Liver transplantation in mainland China: the overview of CLTR 2011 annual scientific report. Hepatobiliary Surg Nutr 2, 188-197

Zhang, Y.C., Qu, E.Z., Ren, J., Zhang, Q., Zheng, R.Q., Yang, Y., and Chen, G.H. (2014). New diagnosis and therapy model for ischemic-type biliary lesions following liver transplantation: a retrospective cohort study. PLoS One 9, 1-7.

Open Access This article is distributed under the terms of the Creative Commons Attribution License which permits any use, distribution, and reproduction in any medium, provided the original author(s) and source are credited. 